\title{
Ontogenic stages for chemical instrumentation
}

\author{
Each successful new measurement tool passes through three stages: first, it is hyped by its creators and \\ ridiculed by threatened competitors; second, it is accepted by a few for refinement and validation; \\ and third, it accelerates to maturity or fades away."
}

Keywords: analytical instrumentation $=$ evolving science tools $\approx$ measurement tools

\section{Evolution of analytical instrumentation}

I am at the stage of life where organ systems draw on some of their redundancies. Repairs to physical insults take much longer now. I am hoping some wisdom has been gained, but one can never be sure. A lot of analytical instrumentation developments have passed in front of me at hundreds of scientific meetings over four decades. A few of these advanced from curiosities to world dominance in our field, others favored less well.

For example, I was extremely fortunate to engage with modern LC while it was still incubating in academia and at DuPont and Waters. That was a wave not to be missed as we entered the 1970s. The new tool solved bioanalytical problems in ways that nothing else at that time could. It was faster. It was easier. It was more accurate and precise. As with an aircraft, you could hang on all sorts of accessories, not missiles, but injection systems, detectors, software, sample preparation robotics and a wide variety of stationary-phase chemistries. In addition, you could also go small or you could go large, picograms to kilograms, and you could go fast or slow, seconds to hours. Steve Jobs liked the description 'insanely great' and that fits only a few bioanalytical advances since World War II. Among them all, LC arguably has had the broadest impact, although others may select NMR, MS or immunoassays. Discussing such comparisons is instructive for students, sharpening their analytical skills in defining the best from among the contenders. There is no right answer. Let us consider the evolution of analytical instrumentation in anthropomorphic terms.

\section{Stage I: birth}

First there is skepticism. Will this new approach provide useful data in the real world? Topics at this early stage today include low-cost, paperbased diagnostics for under-developed economies, carbon nanotube- and graphene-based sensors, as well as a variety of new chip-based arrays that take us beyond genomics.

\section{- Stage 2: childhood}

The community of innovators and early adapters is challenged to prove their enthusiasm is justified. Dried blood spots for regulated quantitative bioanalysis, a favorite of this journal, is in motion to stage 3 . The same for the many microfluidics tools, which have been struggling to breakthrough since the 1990s.

\section{- Stage 3: young adults}

With experience comes significant refinement and then commercial implementation. Ambient ionization MS, in vivo human glucose sensors and surface-enhanced Raman spectroscopy fit this category. The first is a development of the current century. The last two have been incubating for decades.

\section{- Stage 4: middle age}

Commercial products are vetted in the literature with respect to applications, often in regulated sectors such as food, environment, diagnostics and pharmaceutical. Performance improves and older tools are displaced. MALDI-MS, near IR and UHPLC have now reached this stage.

\section{- Stage 5: maturity}

Best practices are established. Publications begin to move to third-tier journals and trade magazines. Representative adults include autotitrators, GC, home-use glucose sensors, gel electrophoresis, capillary electrophoresis, HPLC-UV, ion chromatography, electrospray LC-MS/MS, lateral flow diagnostics, $\mathrm{pH}$, ion selective electrodes, TLC, oxygen electrodes, 2D NMR, cyclic voltammetry,

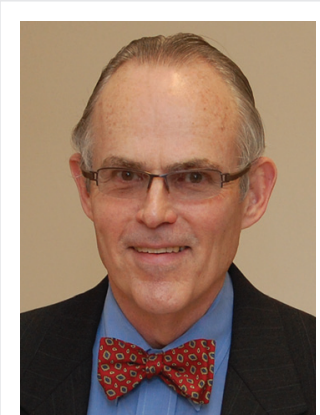

Peter T Kissinger

Purdue University, West Lafayette, IN 47907, USA

E-mail: kissingp@purdue.edu 
atomic absorption, Raman, fluorescence imaging and ICP-MS. At this stage we find a few very broadly applicable success stories, but also many examples where the initial excitement faded, and a few where specific, very compelling applications settled in nicely. Examples of the latter include capillary electrophoresis for genomics, ion selective electrodes for electrolytes in blood, Mössbauer spectroscopy and atomic emission for metals.

\section{Stage 6: critical care \& death}

The history of science takes over. In this category, we have polarography, two pan balances, ion-selective field effect transistors, optrodes, surface acoustic waves, flame photometry, chronopotentiometry, field-desorption MS, post-column reactions in LC and self-assembly of thiols on gold. Much was learned that was of fundamental interest. There were good practical runs for a few before they were displaced. Two pan balances had a solid millennium or two - that's the record holder.

\section{Discussion}

Arthur Schopenhauer noted that "All truth passes through three stages: First, it is ridiculed; second, it is violently opposed; and third, it is accepted as self-evident." I have always liked this quote, but it is limited to newly exposed truth. One may consider this wisdom while debating the now firmly established truths of evolution and climate change. Those things that are not true are not encompassed in Schopenhauer's pithy quote. Let us see how we might paraphrase his thought for chemical instrumentation.

Each successful new measurement tool passes through three stages: first, it is hyped by its creators and ridiculed by threatened competitors; second, it is accepted by a few for refinement and validation; and third, it accelerates to maturity or fades away. The breadth of applications either broadens or narrows from the original conception. For example, those of us of a certain age saw LC dramatically broaden and capillary electrophoresis, supercritical fluid chromatography, biosensors and field-flow fractionation dramatically narrow from original expectations. I can recall days when pharmaceutical companies and the US FDA had very real doubts that LC would ever be competitively rugged versus GC for either formulated drugs or bioanalytical applications. We were at stage $2 / 3$ for LC in the mid-1970s. It was not clear then that two LC columns would be sufficiently similar to justify a quality-regulated method. At the time, coupling reversed-phase LC to a mass spectrometer was impossible, a combination that was not meant to be.

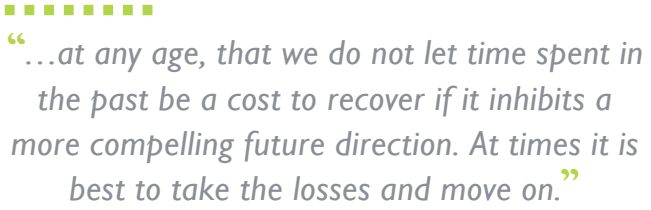

It is clearly a challenge to predict those approaches that will survive to maturity and either have a broad impact or be insanely great in one very narrow application. Knowing when to quit is also a challenge. I see many cases where we try too hard and for too long, not moving out of our peer-reviewed tribe. Other concepts narrowly escape death with new insights. TOF-MS comes to mind. My advice to young scientists: read and think broadly, move out of your comfort zone, and look forward not backwards. I suggest, at any age, that we do not let time spent in the past be a cost to recover if it inhibits a more compelling future direction. At times it is best to take the losses and move on.

\section{Financial \& competing interests disclosure}

The author has no relevant affiliations or financial involvement with any organization or entity with a financial interest in or financial conflict with the subject matter or materials discussed in the manuscript. This includes employment, consultancies, honoraria, stock ownership or options, expert testimony, grants or patents received or pending, or royalties.

No writing assistance was utilized in the production of this manuscript. 